\title{
Thermal characterization of multi-layer polymer films by IR thermography
}

\author{
by J. Morikawa*, T. Hashimoto*, E. Hayakawa**, T. Eto***, and R. Li Votiं ${ }^{* * *}$ \\ *Tokyo Institute of Technology, Tokyo, Japan \\ **ai-Phase, Co., Ltd., Tokyo, Japan \\ ***Eto Denki, Co., Ltd., Tokyo, Japan \\ ****University of Rome, Rome, Italy
}

\begin{abstract}
The measurement of thermal wave propagating in the cross section of polymer film layers including the interfaces was performed by edge view observation of IR thermographs analyzed by the Fourier- transformed phase image. The phase jumps observed experimentally, indicates the presence of a thermal boundary resistance between each layer. This effect has been modelled by introducing thin surface skins of poor conductors (air, grease) among polymeric layers. The numerical analysis allows estimating the thickness of these skin layers of less than 3 $\mu \mathrm{m}$.
\end{abstract}

\section{Introduction}

To design and control the thermal interface is one of the important technology especially in industry in view of the efficient power control. The methodology and experimental technique to quantify and determine the thermal characterization of the interface is still under discussion. In this study the thermal characterization of the cross section of polymer film layers including the interface was performed by an edge view observation of IR thermographs and the Fourier- transformed phase image. An analytical simulation of thermal wave in multi layers assuming the thermal impedance on the interface was applied to analyse the thermal characterization of the interface.

\section{Experimental}

Phase shift analysis of temperature wave is often used to determine thermal diffusivity of thin film. Temperature wave analysis [1] is one of the methods to determine thermal diffusivity in one-dimensional (1D) geometry. In this study IR FPA system combined with TWA is set up for quantifying the thermal interface with the observation of the phase shift.

\subsection{IR (Infra red) FPA (Focal plane arrays) system}

InSb FPA (Phoenix, Indigo Systems, and Radiance HS, Raytheon) was used which is sensitive in $3 \sim 5 \mu \mathrm{m}$ spectral band. The array of $256 \times 256$ pixels and IR optics allowed $1.9 \times 1.9 \mathrm{~mm}^{2}$ specimen area giving a spatial resolution of $7.5 \mu \mathrm{m} \sim 3 \mu \mathrm{m} / \mathrm{pixel}$ with SiGe micro lens (ai-phase Co., Ltd.). The frame rate varied from $60 \mathrm{~Hz}$ to $5000 \mathrm{~Hz}$, corresponding to the pixel size $256 \times 256 \sim 64 \times 64$. The time resolved IR photograph of the temperature wave propagating in the specimen was obtained in the most suitable conditions of the frame rate and the shutter speed, and 
simultaneously the time profiles of temperature at all pixels were extracted. The direct access system to Hard disk was originally prepared for the longer time data storage. The measurement was performed both in the atmospheric and the reduced pressure. The propagation of the thermal wave on the top surface of the cross section of the specimen was observed by InSb FPA cooled at 77K with SiGe micro lens through the window of the sapphire glass of the vacuum chamber (Fig. 1).

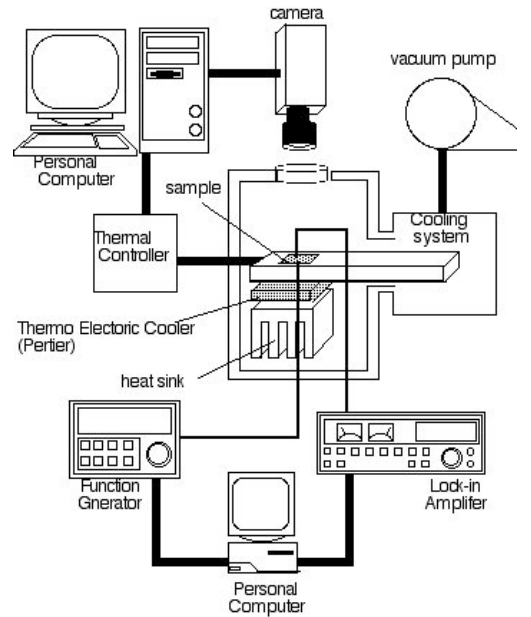

Fig.1. Schematic diagram of experimental setup for IR FPA with TWA system[2].

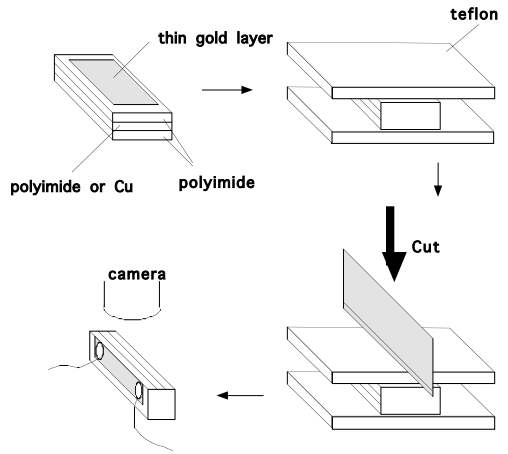

Fig. 2 Preparation of the cross section of layered film.

\subsection{Multi layer films}

The cross section of the three layered polyimide films was prepared as shown in Fig. 2 on which the propagation of thermal waves were observed. The thickness of each layer is $125 \mu \mathrm{m} / 75 \mu \mathrm{m} / 125 \mu \mathrm{m}$ and the interfaces between the films were filled with (and without) the thermal grease compounds having the known thermal properties. On the outside surface of the outside layer a gold thin layer was sputtered, which was used as a heater generating a temperature wave by a.c. Joule heating with a function synthesizer (NF1942) in the range of $0.01 \mathrm{~Hz}$ to $10 \mathrm{~Hz}$. The geometry of the interfaces and the heater has both a plane symmetry (1D geometry).

\subsection{Temperature wave analysis (TWA) system}

TWA[1] is a method to determine the thermal diffusivity of thin films measuring the phase shift between the top and the rear surfaces by using a sputtered metal layer as a temperature sensor. The gold layers were sputtered on the both surfaces of the multi layer films in this study. The simultaneous measurement of the total phase shift in the multi layers were determined by using a digital lock-in amplifier (SR830).

\section{Analytical method}

2.4. $\quad 3.1 \quad$ Fourier transformed phase image of IR thermography The time resolved IR thermograph was analyzed at each pixel with Discrete Fourier transform,

$$
G(\mathrm{n} / \mathrm{N})=\frac{1}{\mathrm{~N}} \sum_{\mathrm{k}=0}^{\mathrm{N}-1} \mathrm{~g}(\mathrm{k}) \exp (-\mathrm{j} 2 \pi \mathrm{nk} / \mathrm{N})
$$


Where $G(n / N)$ is a discrete Fourier transform function, $N$ is a sample number in one cycle, and $f=1 / N$ is frequency. The picture image of phase is taken by the difference with the pixel on which the temperature wave is generated.

\section{5. $\quad 3.2$ Mathematical theory of thermal wave at the thermal}

\section{interface}

In case of a multilayer the plane thermal wave, propagating in the layered structure, is subjected to a multiple reflection phenomenon. In general $i^{\text {th }}$ layer the following thermal wave interference takes place where the backward wave is included

$$
\hat{T}_{i}(z)=A_{i} \cdot \exp \left[-\beta_{i} z\right]+B_{i} \cdot \exp \left[\beta_{i} z\right],
$$

where $\beta_{i}=\left(j \omega / D_{i}\right)^{1 / 2}$ is the wave number of the $i^{\text {th }}$ layer, and the coefficients $A_{i}$ and $B_{i}$ should be determined by the boundary conditions at each interface.

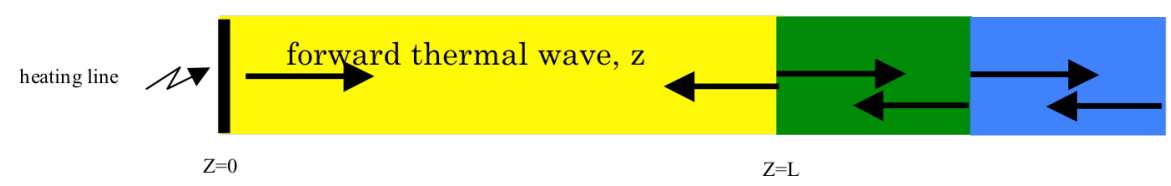

Fig. 3 Schematic model of multi layer and the thermal wave.

The thermal wave reflectivity is defined as the ratio between the backward and forward thermal wave, which is described as follows.

(2)

$$
\Gamma_{i}(z)=\frac{B_{i} \exp \left[\beta_{i} z\right]}{A_{i} \exp \left[-\beta_{i} z\right]}=\frac{B_{i}}{A_{i}} \exp \left[2 \beta_{i} z\right]=\Gamma(0) \exp \left[2 \beta_{i} z\right]
$$

Inside the layer the quantity $\Gamma(z)$ is $z$ dependent. In particular for a layer of thickness $L$ we may calculate the reflectivity at $z=0$ with respect to the reflectivity at $\mathrm{z}=\mathrm{L}$ as follows

(3)

$$
\Gamma_{i}(0)=\Gamma(L) \exp \left[-2 \beta_{i} L\right]
$$

By simplifying the temperature profile in Eq.(1) with the use of the thermal reflectivity and by assuming the continuous temperature and heat flux at the $i^{\text {th }}$ interface, one may calculate the relationship between $\Gamma\left(0^{-}\right)$and $\Gamma\left(0^{+}\right)$as shown in Eq.(4).

(4)

$$
\begin{gathered}
\Gamma_{i}\left(0^{-}\right)=\frac{\Re_{i, i+1}+\Gamma_{i+1}\left(0^{+}\right)}{e_{i} 七 \Re_{i+1+1} \cdot \Gamma_{i+1}\left(0^{+}\right)} \\
\Re_{i, i+1}=\frac{e_{i+1}}{e_{i}+e^{2}}
\end{gathered}
$$
follows.

where $\mathrm{R}$ an intrinsic reflectivity at the interface, that has been introduced as 
In a simple example, such as the case in which the structure of a film over a semi infinite bulk, the temperature in the film ( $1^{\text {st }}$ layer) is derived as

(5)

$$
\hat{T}_{\text {film }}(z)=\frac{I}{e_{\text {film }} \sqrt{j \omega}} \cdot \frac{\exp \left[-(1+j) \frac{z}{\mu_{\text {film }}}\right]+\Re_{1,2} \exp \left[-(1+j) \frac{z-2 L}{\mu_{\text {film }}}\right]}{1-\Re_{1,2} \exp \left[-2(1+j) \frac{L}{\mu_{\text {film }}}\right]}
$$

It is interesting to show the phase plot of the temperature in the film layer as a function of $z$ and for different values of the intrinsic reflectivity. For example the calculation results are shown with the thickness of the film about $L=100 \mu \mathrm{m}$ and the thermal diffusion length also at $\mu=100 \mu \mathrm{m}$ in order to see the effect of the thermal interference.

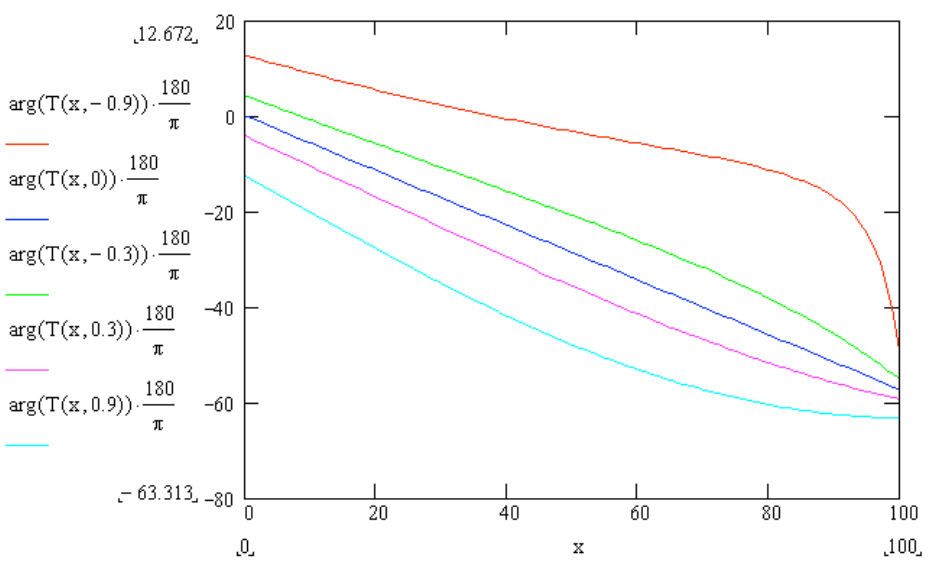

Fig. 4 Numerical simulations of phase shift $\Delta \theta$ of temperature wave in the schematic model in Fig. 3 with different value $R$ of intrinsic reflectivity; red : $R=-0.9$, green : $R=-0.3$, purple : $R=0$, pink $: R=0.3$, blue $: R=-0.9$.

The blue curve represents the case of $R_{12}=0$, when bulk and film have the same thermal effusivity. In this case the phase is still linear as for a homogeneous case. But if the bulk is more (less) effusive than the film $R<0(R>0)$ the phase exhibits some distortion from linearity. By assuming the five-layer mode the numerical fitting with the experimental phase data is examined to apply the reflection of temperature wave at the interface.

\section{Experimental results}

4.1 Phase and amplitude image of temperature wave

The snap shot from the time resolved IR thermographs of the top view of the three layered polymeric films is shown in Fig. $5 a$, where the thermal wave is 


\section{http://dx.doi.org/10.21611/qirt.2006.020}

generated on the outside surface of the left $1^{\text {st }}$ layer. The color shows the intensity of the photon counting by FPA in arbitral unit with 12 bit digital signal that is quantified by the temperature of the certified reference materials measured in the same condition. The propagation of temperature wave from the $1^{\text {st }}$ layer to the $2^{\text {nd }}$ and the $3^{\text {rd }}$ is clearly observed as the gradient of the color. The thermal image of waveform of $1 \mathrm{~Hz}$ and its Fourier transform spectrum in a center pixel of the 1st layer (shown as the red cross point) are also shown in Fig.5a in the ambient pressure. Mathematics predicts that thermal wave decays with the phase delay in the $1 \mathrm{D}$ propagation. In Fig. 5 that is directly observed by FPA. Fig. $5 \mathrm{~b}$ shows that is observed at $0.2 \mathrm{~Hz}$. The difference of the temperature gradient means the difference of $1 \mathrm{D}$ thermal diffusion length. In Fig. 5 a\& b the surface inhomogeneity is still observed.

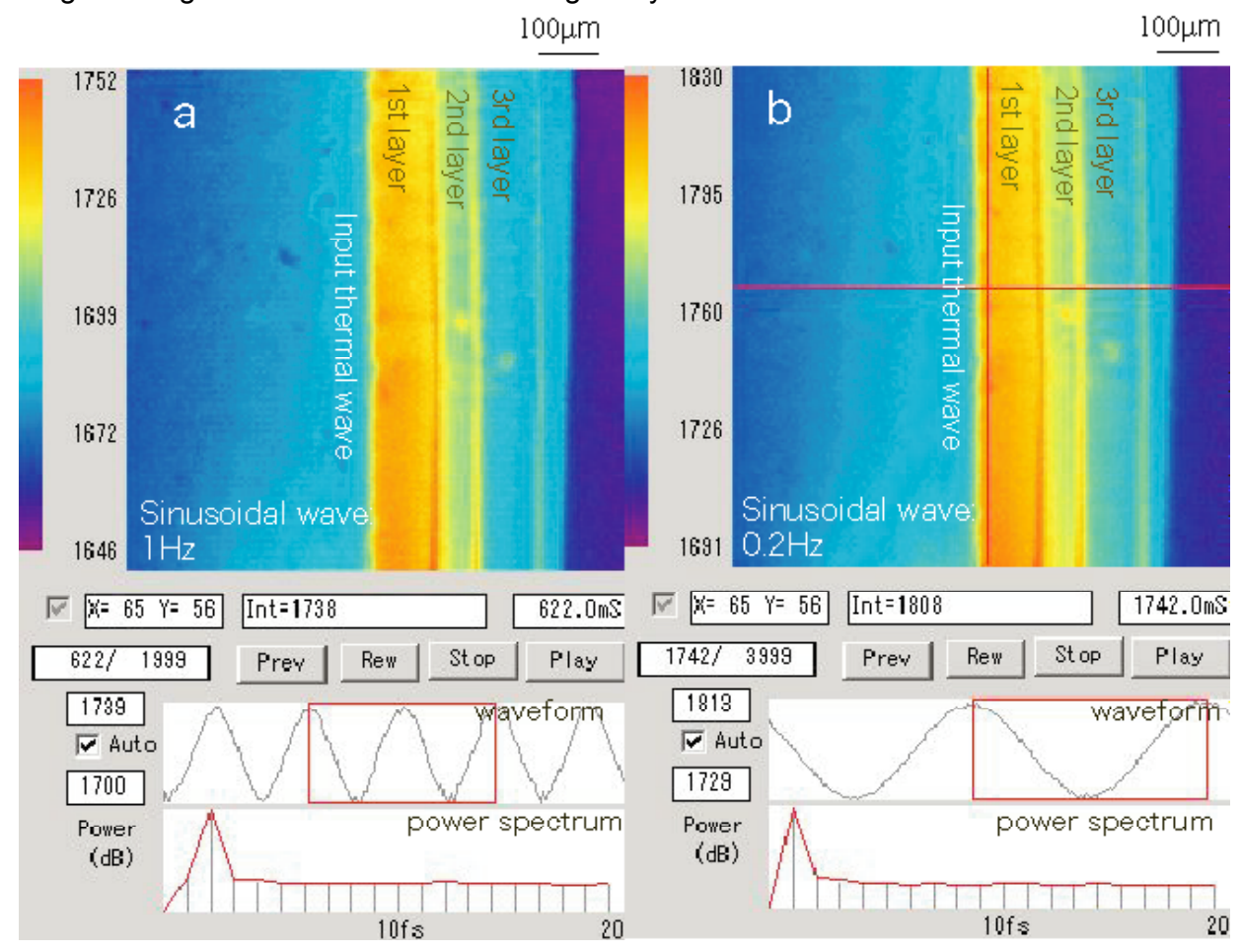

Fig. 5 Thermal image of the cross section of the three layers polymide films with $125 \mu \mathrm{m} / 75 \mu \mathrm{m} / 125 \mu \mathrm{m}$ input with the sinusoidal temperature wave of a) $1 \mathrm{~Hz}$, and b) $0.2 \mathrm{~Hz}$ observed by the IR FPA with the microscopic lens in the area size of $1.9 \mathrm{~mm}$ $x 1.9 \mathrm{~mm}$ with the pixel size of $7.5 \mu \mathrm{m}$. The waveform and the power spectrum are also shown.

Fig. 6a d are the Fourier transformed phase and amplitude images at different frequencies. In these figures the arc. temperature components are extracted. The 1D geometry of thermal wave propagation allows the 1D phase and amplitude gradients from the $1^{\text {st }}$ to $3^{\text {rd }}$ layers on the direction of the horizontal line. 

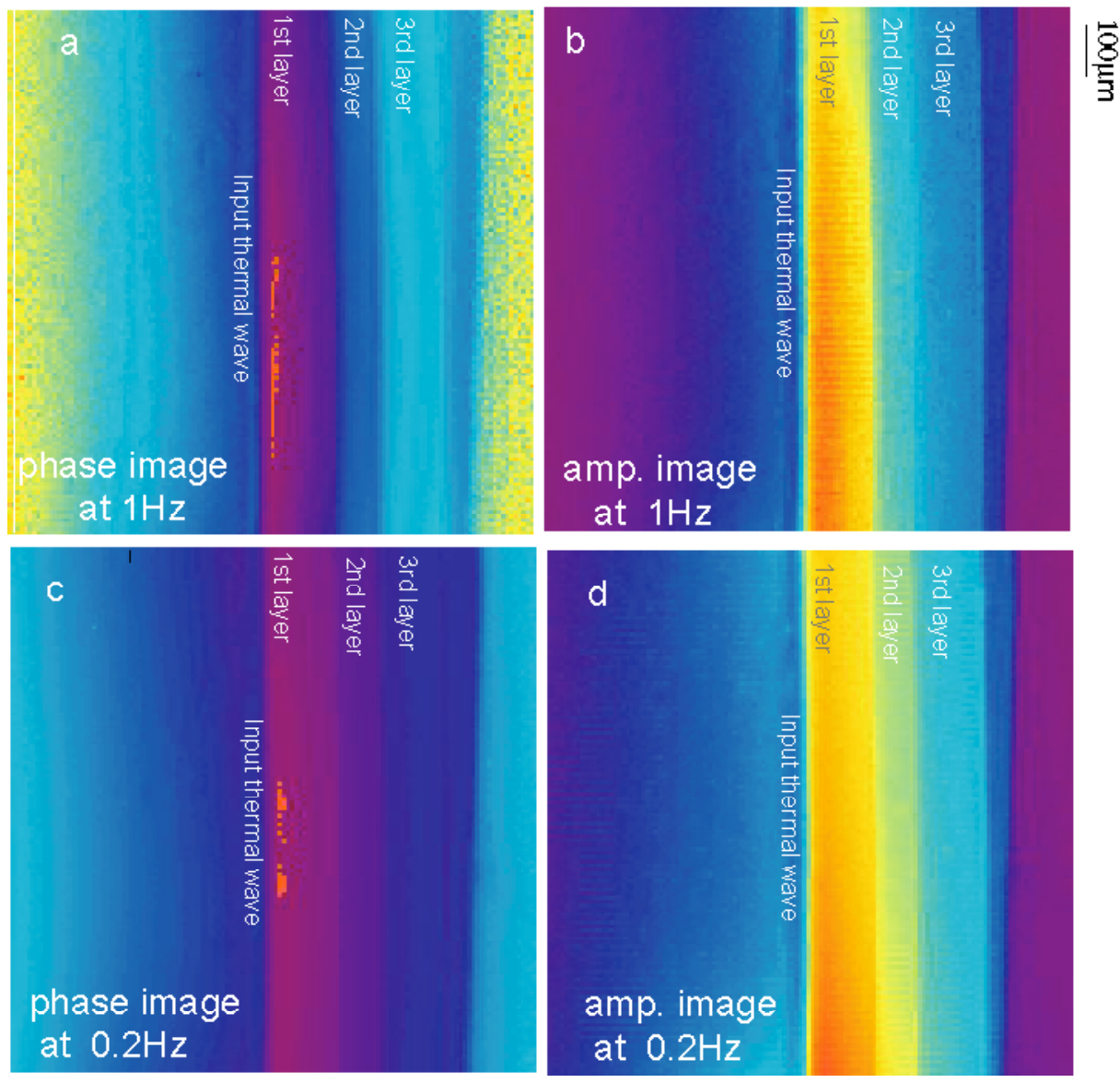

Fig. 6. Fourier transformed phase image and amplitude image of three layers of polyimide films without any thermal greases at the interface at different frequencies; a) $1 \mathrm{~Hz}$ phase image, b) $1 \mathrm{~Hz}$ amplitude image, c) $0.2 \mathrm{~Hz}$ phase image, and d) $0.2 \mathrm{~Hz}$ amplitude image. The colour in the phase image is drawn in the range of $0 \sim \pi$. the dark colour corresponds to zero; the bright colour corresponds to the larger phase delay. The colour in the amplitude image b) and d) is comparatively attributed in the auto gain control. The input thermal wave source is on the left surface of the $1^{\text {st }}$ layer.

\subsection{Phase profile across the layers}

The phase profile with different interface conditions are plotted in Fig. 9-1 in the atmospheric pressure. The thermal conditions are; with air (no thermal grease) $\mathrm{R}=0.98$, with grease $A$ (apiezon) $\mathrm{R}=-0.17$, grease $\mathrm{B}$ (San Hayato $\mathrm{SCH} 30$ ) $\mathrm{R}=-0.42$, 
and grease $C$ (Shinetsu K913) $R=-0.53$. In Fig. 9-1 the right side of the layers is a position of input power source. Between the layers phase jumps are observed in Fig. 9-1(a-d), however the slopes show the difference with the different interface conditions. The relationship between the intrinsic reflectivity $R$ and the phase jump at the interface is complicated, but totally in three layers the larger absolute value of $R$ corresponds to the increase of the total phase delay, indicating the distortion of the slope in the plot of $\Delta \theta$ vs. $d$ including the phase jump. These results suggest that the complicated thermal transfer occurs at the interface, including the thermal wave reflection or thermal conductance
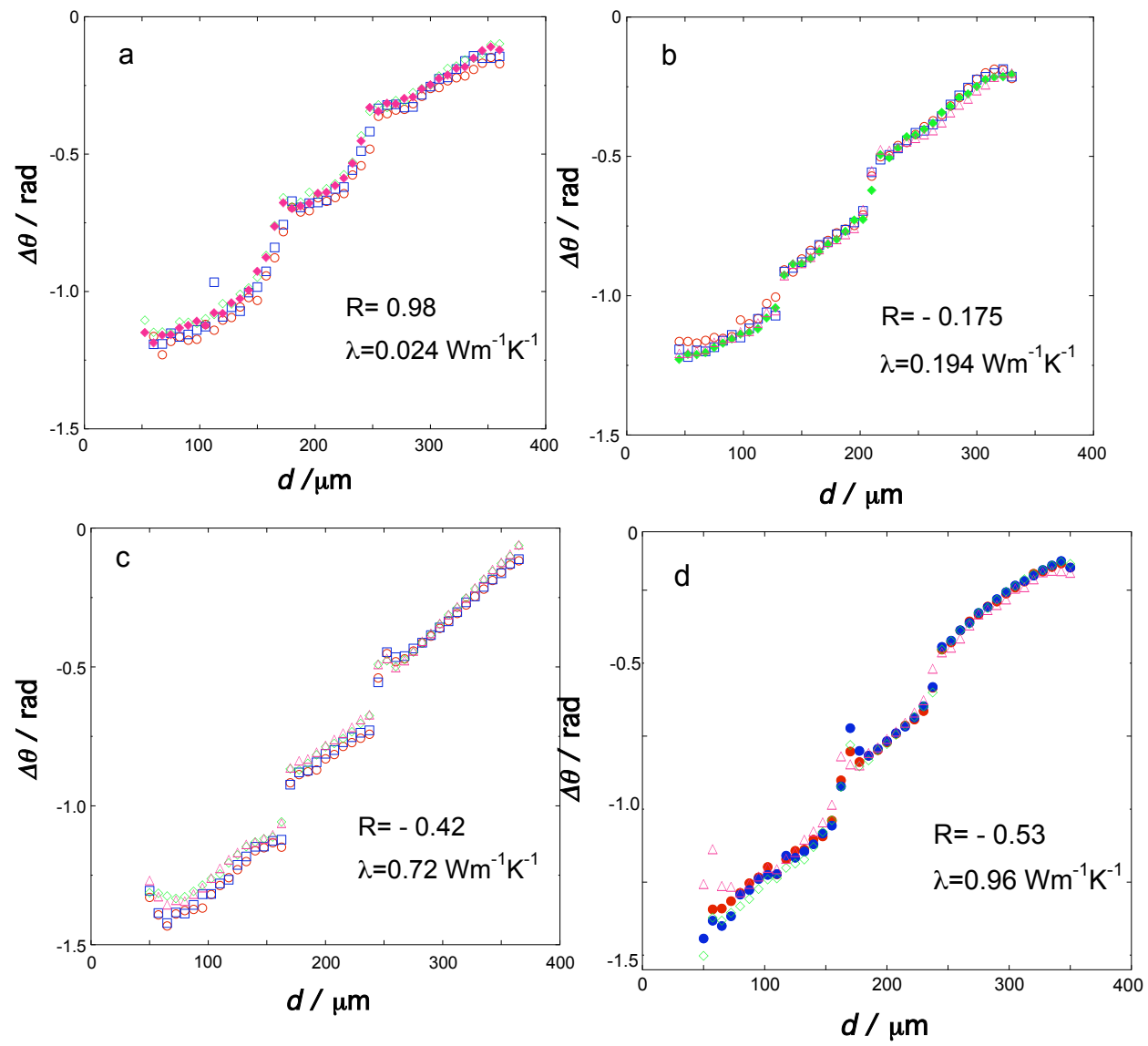

Fig. 9-1. Phase profile across the three layers observed at $1 \mathrm{~Hz}$ in the ambient pressure with various reflectivities at the interface. A) $R=0.98, b) R=-0.17, c$ ) $R=-0.42, d) R=-0.53$.

In Fig. 9-2a $\mathrm{R}$ dependence on the phase shift at the interface in the three layered film observed by TWA measurement is shown. The interface phase shift becomes larger with the larger absolute value of $R$ that agrees the results obtained in the phase profiles in thermographs. Fig. $9-2 b$ shows the relationship of $R$ and the thermal conductivity of the thermal grease at the interface. Higher the thermal 
conductivity, higher the phase shift at the interface is obtained. This result means the importance of the thermal reflection at the thermal interface.
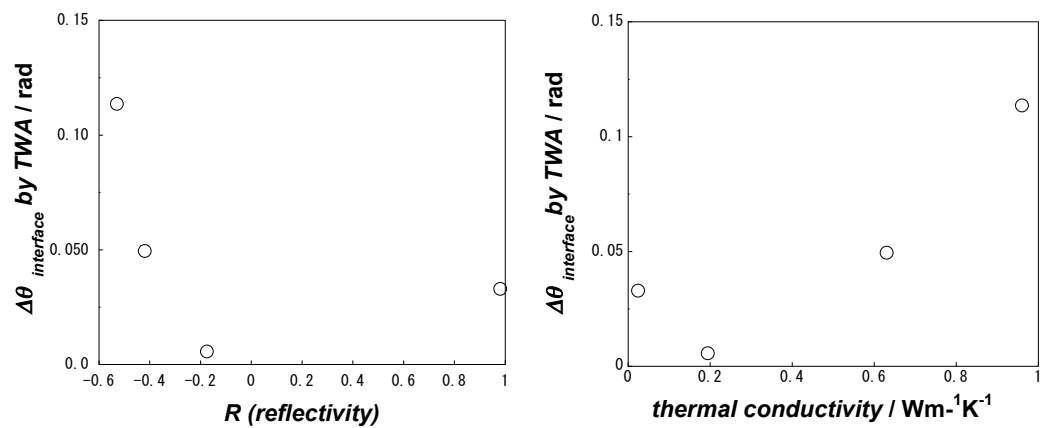

Fig. 9-2. $\quad$ Phase delay at the interface determined by the TWA method. a) plotted against reflectivity $R, b)$ plotted vs. thermal conductivity of the thermal grease in the interface

The phase profile across the layers observed in the reduced pressures with no thermal grease condition, the phase jump is also observed at the interface and the phase slope becomes sharp than observed in the atmospheric pressure. It suggests that the influence of the convection with the surface air is to be taken into account.

\section{Discussion of the numerical calculations}

The experimental data of phase shift have been compared and fitted by appropriate numerical simulations of the thermal field in the multilayered polymeric samples such as shown in Eq. (5). The phase jump observed experimentally, indicates the presence of a thermal boundary resistance between each layer.

This effect has been modelled by introducing thin surface skins of poor conductors (air, grease) among polymeric layers. The numerical analysis allows estimating the thickness of these skin layers of less than $3 \mu \mathrm{m}$ (Fig.10).

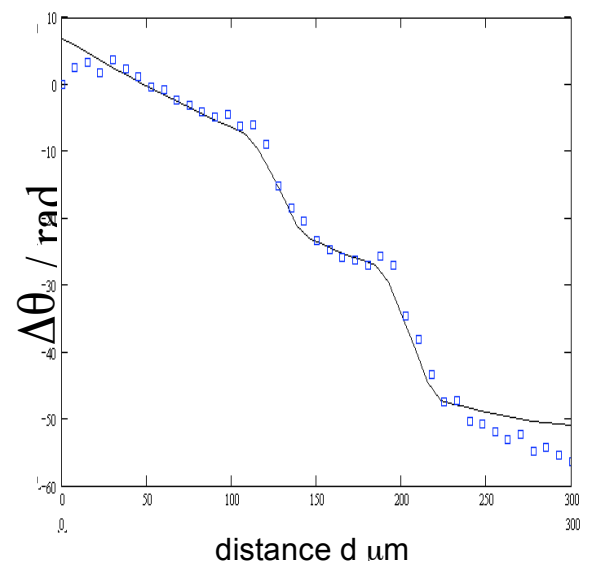

Fig. 10. Numerical simulation (straight line) and the experimental results (circles) of phase shifts $\Delta \theta$ of temperature wave at $1 \mathrm{~Hz}$ in the three layered films. 


\section{Conclusion}

The method of direct view of thermal interface by IR thermographs is proposed in this paper. The thermal interface of three layers of polymer film, including the air or the thermal grease with the different reflectivity/thermal conductivity, is analyzed with the phase image in the 1D geometry. The phase profile shows the jump at the interface and the distortion of the slope. The mathematical model considering the thermal reflection at the interface fits the phase profile in the atmospheric pressure condition.

\section{References}

[1] J. Morikawa, T. Hashimoto Jon. J. Apple. Phys., 37, L1484 (1998).

[2] J.Morikawa, T.Hashimoto, E.Hayakawa, H.Uemura, Thermosense XXV SPIE 5073, 148 (2003).

[3] M.Bertolotti, M.Firpo, R.Li Voti, S.Paoloni, C.Sibilia, F.Tani and G.L.Liakhou, Progress in Natural Science, 6, 219 (1996). 